\title{
Telemetry Data Analysis on sEMG Major Muscle Groups in Curving-Round-Bridge-Posts of 400m Obstacle
}

\author{
Xiaonan $\mathrm{Wu}^{*}$, Bo Zhang, Mei Song \\ Information Communication College, National University of Defense Technology, Xi'an Shaanxi 710106, China \\ *Corresponding author. Email: 515734324@qq.com
}

\begin{abstract}
Objective: The purpose of the study is to explore the sEMG characteristics of associated major muscle groups (agonist muscles) in curving-round-bridge-posts event of 400m obstacle and its relationship with muscle performance. Methods: This study tested seven male military cadets who did quite well in $400 \mathrm{~m}$ obstacle, and analyzed the original electromyography, iEMG and iEMG\% data by using the surface electromyography telemetry and anatomical analysis of the action, combined with sEMG synchronal video data analysis. Results: The power order of the muscle strength in left upper limb and trunk muscle groups is: all test muscle groups are almost synchronized muscle power; the power order of the left lower limb muscle group and rectus abdoninis in sequence is: rectus abdoninis and tibialis anterior first generate power, followed by quadriceps, hamstrings, gastrocnemius, and gluteus maximus in sequence. The order of iEMG and iEMG\% from large to small is: the left upper limbs and trunk muscle groups (biceps brachii muscle, latissimus dorsi muscle, triceps muscle of arm, trapezius muscle, wrist flexors, deltoid muscle, pectoralis major muscle, and wrist extensors); the left lower limb muscle and rectus abdoninis (rectus abdoninis, hamstrings, gluteus maximus, quadriceps, gastrocnemius, and tibialis anterior). Conclusion: In the process of curving round the bridge posts, the key action is to rely on upper limb and shoulder-back power to get the body close to the bridge post to control inertial deviation. The left arm should be pull out as soon as possible to avoid motion delay, and to make sure the body may approach the bridge post as close as possible and move rapidly along the shortest route. In teaching and training of the curving-round-bridge-posts activity, great efforts should be made to develop the muscle strength of the upper limb biceps, brachialis, triceps muscle of arm, wrist flexors, latissimus dorsi muscle and trapezius muscle; focus should also be put on improving the muscle strength of the lower limbs hamstrings, gluteus maximus, quadriceps, triceps surae, and rectus abdoninis.

Keywords: 400m obstacle, curving-round-bridge-posts event, surface electromyography telemetry, original
\end{abstract} electromyography, iEMG, iEMG\%

\section{INTRODUCTION}

$400 \mathrm{~m}$ obstacle is the traditional key training and test event in military sports, which is a series of different types of obstacles simulated in wartime[1]. The training event consists of certain skillful actions including: free running, jumping, climbing, crawling, grabbing, pulling, hanging and crossing. It is vital to study the features of muscle power in the movement of curving-round-bridge-posts to enhance the power capability of overcoming obstacles. The research intends to provide reference in action techniques and muscle power improvement in $400 \mathrm{~m}$ obstacle training by analyzing telemetry dada on sEMG major muscle groups in curving-round-bridge-posts event.

\section{SUBJECTS AND METHODS}

\subsection{Subjects}

7 male cadets from a military academy, who were performed with standard movement of actions in $400 \mathrm{~m}$ obstacle and the time limit of $11^{\prime} 55^{\prime \prime}$, healthy, average age $(21.86 \pm 1.07) \mathrm{y}$, average height $(174.86 \pm 4.88) \mathrm{cm}$, average weight $(64.12 \pm 5.15) \mathrm{kg}$. 


\subsection{Methods}

\subsubsection{Action techniques analysis}

Running to the left (or right) side of the first bridge post, knees bent, the right (or left) foot stepping out to the right (or left) side of the second bridge post, with the left (or right) hand holding the second bridge post, and the body

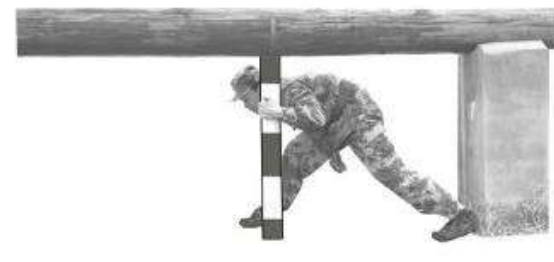

A

Figure 1 Movement of Curving-round-bridge-posts

\subsubsection{Action node and time period of sEMG test}

Picture 1 shows: Node A: knees bent, the right foot moving forward, the left hand holding the bridge post, and then the left foot moving forward; Node B: after the left foot touching the ground, the right hand holding the bridge post, the right foot are ready to move on. The key muscle power actions in the movement from $\mathrm{A}$ to $\mathrm{B}$ include: holding the bridge posts with upper arms to control the body, touching the ground with the right or left foot, moving forward from the same-side.

\subsubsection{Muscle test design}

Movement techniques of curving around the right or left side of the bridge posts were almost the same. Based on the movement analysis of anatomy, and the measurability of sEMG, 14 muscles were chosen to be tested in upper limbs, trunk muscle groups, and lower limb muscles. leaning inside against it; the right (or left) foot moving round the second bridge post and then toward the left (or right) side of the third bridge post, with the right (or left) hand holding the third bridge post, and the body leaning inside against it; the right (or left) foot moving round the third bridge post, and turning right (or left) side, running on, and then coming out of the bridge (Picture 1) [1]

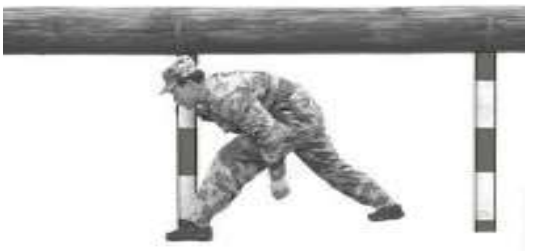

B

According to movement features from $\mathrm{A}$ to $\mathrm{B}$, and 8-channel measurability of electromyography, the 14 muscles were completed in two tests. The first test (left side): deltoid muscle, biceps brachii muscle, triceps muscle of arm, wrist flexors, extensor muscle of wrist, trapezius muscle, latissimus dorsi muscle, pectoralis major muscle; the second test (right side): rectus abdoninis, gluteus maximus, quadriceps, hamstrings, tibialis anterior, gastrocnemius.

\subsubsection{Device and placement of surface electrodes}

8-channel DELSYS Trigno Lap produced in USA was used to get sEMG dada (Picture 2). Signal frequency $2000 \mathrm{~Hz}$, input impedance less than $10 \mathrm{G} \Omega$, noise level less than $0.75 \mu \mathrm{v}$,band pass filtering $(20 \sqcup 450) \mathrm{Hz}$. Video device, produced by Shen Zhen Jin-Yang Technology Co. Ltd., was synchronized with the computer.

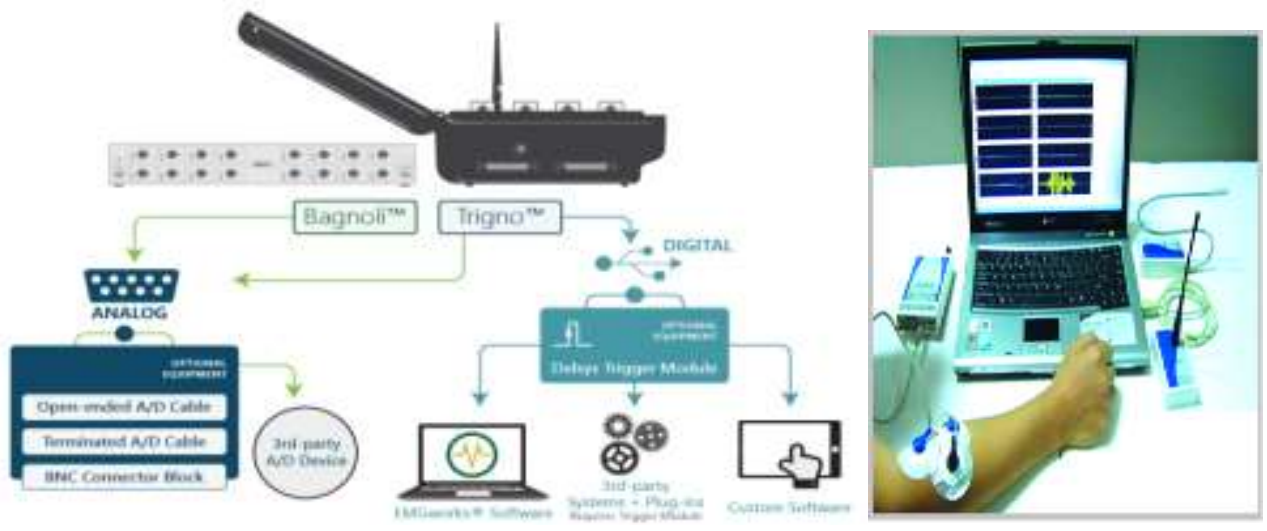

Figure 2 sEMG testing system 
Body hair of the tested muscle part was removed before test, and the skin in which the electrodes were placed was also cleaned by crocus paper and $75 \%$ alcohol sponge to avoid interference to sEMG. The electrodes were placed in the highest point of the tested muscles, with electrode indicating arrow pointing at the direction of muscle fibers, and were fixed by medical adhesive plasters.

\subsubsection{Text index}

\subsubsection{Original electromyography.}

Original electromyography was obtained from unprocessed sEMG, and could be used to identify activation of muscles, duration of muscle functioning, strength of myoelectricity, and coordination mode of muscles[2]. Amplitude of electromyogram showed the amount of muscle power in movement and activation of it. The greater the amplitude of vibration was, the more amount the muscle power were functioning, the more active the muscles were, and the greater contraction power of muscles had[3]. Synchronization analysis was used to directly get 8.0 Signal Analysis results.

\subsubsection{2. $i E M G$ and $i E M G \%$.}

Integral of the electromyography curve of the electromyography and the wrapped area between the transverse axis of the time, was iEMG (unit in $\mu \mathrm{V} . \mathrm{S}$ ). iEMG reflected the strength of muscle's electromyoelectric activity during the selected time period, and, to some extent, the amount of muscle power throughout the movement[4].

The percentage of the total iEMG value of a test muscle for all test muscles involved in the action was the contribution rate (iEMG\%) for that muscle; iEMG\% showed the proportion of the muscles measured in completing the movement[5,6].

\subsection{Data Processing and Statistical Analysis}

Capture video synchronous analysis software, developed by Beijing Century Tianhong in the Labview environment, was used to process sEMG data. Because each experimental subject had a different time per test, the MATLAB software was used to conduct each test time $t_{1}$, $\mathrm{t}_{2}, \mathrm{t}_{3}, \ldots \mathrm{t}_{\mathrm{s}}$ for maximum normalizing, i.e. $t_{i}^{*}=\frac{t_{i}}{\max \left\{t_{1}, t_{2}, \ldots t_{s}\right\}}, 1 \leq i \leq s$. After the time normalized processing, iEMG was obtained. iEMG was statistically processed by Excel2007, and the data results were represented in $\bar{x} \pm s$.

\section{RESULTS}

\subsection{Results of Original Electromyography, iEMG and $i E M G \%$ in A-B Process in the Left Side Muscle Groups: Deltoid Muscle, Biceps Brachii Muscle, Triceps Muscle of Arm, Wrist Flexors (Extensors), Trapezius muscle, Latissimus Dorsi Muscle, and Pectoralis Major Muscle}

The results of the original electromyogram showed (Picture 3) the muscle power sequence in A-B process was: all test muscle groups were almost synchronized power. From the amplitude, it could be observed (Picture 3 ) that sEMG activation degree is most obvious in deltoid muscle, biceps brachii muscle, triceps muscle of arm, wrist flexors, trapezius muscle, and latissimus dorsi muscle; but sEMG is very weak and discontinuous in wrist extensors, and pectoralis major muscle. The biggest sEMG activation degree appeared in the first period from A to B in biceps brachii muscle, triceps muscle of arm, wrist flexors, and latissimus dorsi muscle, and it became weak; the sEMG in the middle period from A to B reached the peak in deltoid muscle and trapezius muscle, and then became weak. iEMG and iEMG\% order from large to small in order: biceps brachii muscle, latissimus dorsi muscle,triceps muscle of arm, trapezius muscle, wrist flexors, deltoid muscle, pectoralis major muscle, wrist extensors (Table 1).

\subsection{Results of Original Electromyography, iEMG and $i E M G \%$ in A-B Process in the Left Side Muscle Groups: Rectus Abdoninis, Gluteus Maximus, Quadriceps, Hamstrings, Tibialis Anterio, and Gastrocnemius}

The results of the original electromyogram showed (Picture 4) the muscle power sequence in A-B process was: rectus abdoninis and tibialis anterior took the lead in synchronous muscle power, and quadriceps, hamstrings, gastrocnemius and gluteus maximus in turn. From the amplitude, it could be observed (Picture 4) that quadriceps maintained a small vibration at high frequency in the beginning sEMG period from $\mathrm{A}$ to $\mathrm{B}$, and disappeared, and then a small electromyoelectric vibration signal was repeated until it was approaching point B. Gluteus maximus and quadriceps in the rear sEMG period from $\mathrm{A}$ to $\mathrm{B}$ began to appear high frequency small vibration, hamstrings in the rear sEMG period from A to B presented explosive discontinuous high frequency vibration; the full sEMG in gastrocnemius was weak; there was a brief period of short-term high-frequency small sEMG in the middle and rear process from A to B respectively. iEMG 
and $\mathrm{iEMG} \%$ order from large to small in order: rectus abdoninis, hamstrings, gluteus maximus, quadriceps, gastrocnemius, tibialis anterior (Table 1).
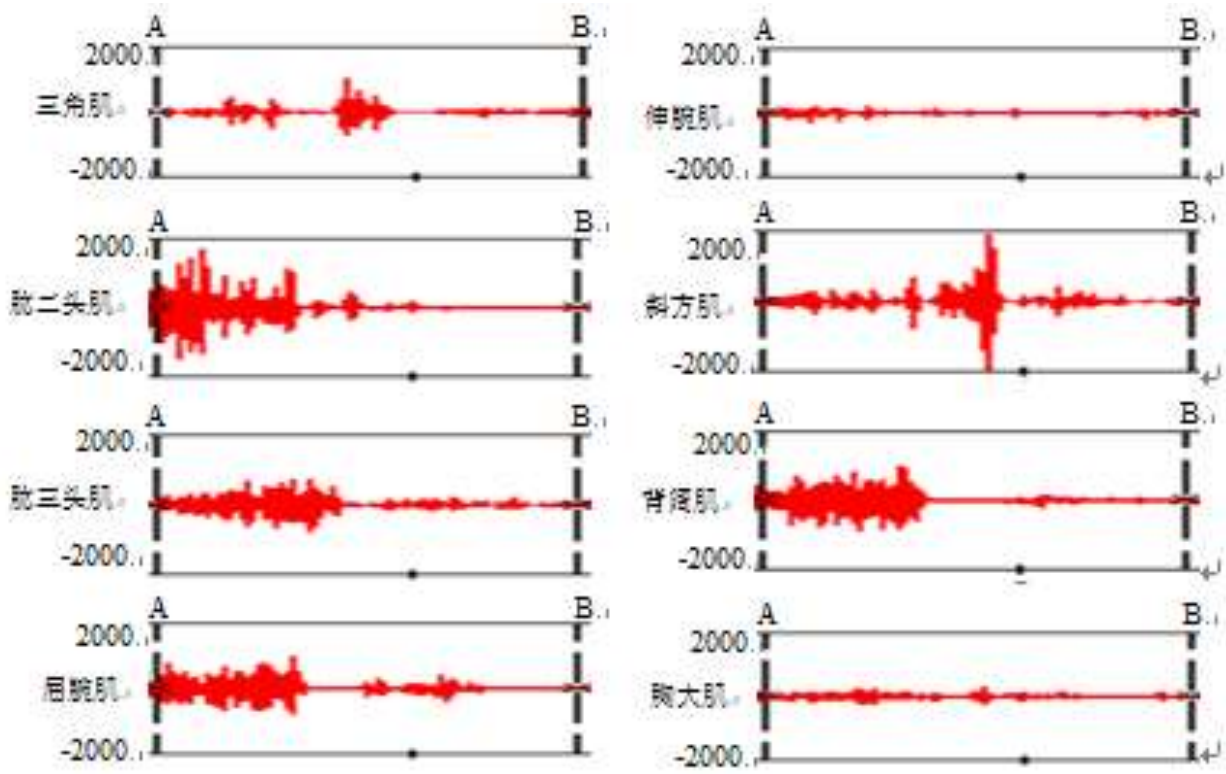

Figure 3 Original Electromyogram in A-B Left Side : deltoid muscle, biceps brachii muscle, triceps muscle of arm, wrist flexors (extensors), trapezius muscle, latissimus dorsi muscle, and pectoralis major muscle
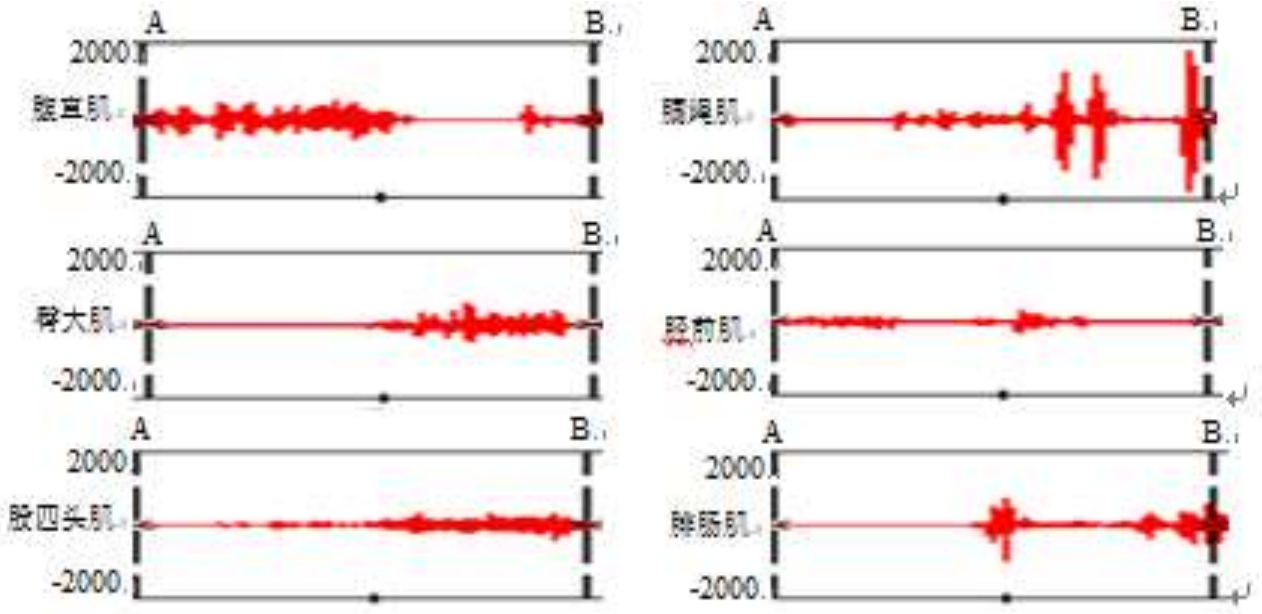

Figure 4 Original Electromyogram in A-B Left Side: rectus abdoninis, gluteus maximus, hamstrings, tibialis anterior, and gastrocnemius

Table 1 Muscle Groups iEMG and iEMG\% $(\mathrm{n}=7) \quad \bar{x}+s$

\begin{tabular}{ccccc}
\hline Side & Muscle Groups & $\begin{array}{c}\text { A-B Process } \\
\text { iEMG }(\mu \mathrm{V} . \mathrm{s})\end{array}$ & $\begin{array}{c}\text { A-B Process } \\
\text { Rate } \%\end{array}$ & $\begin{array}{c}\text { A-B } \\
\text { Process } \\
\text { Order }\end{array}$ \\
\hline \multirow{2}{*}{ Left } & $\begin{array}{c}\text { deltoid muscle } \\
\text { biceps brachii } \\
\text { muscle }\end{array}$ & $100.82 \pm 7.09$ & $8.49 \%$ & 6 \\
& $\begin{array}{c}\text { triceps muscle of } \\
\text { arm }\end{array}$ & $85.30 \pm 10.21$ & $18.59 \%$ & 1 \\
& wrist flexors & $75.36 \pm 7.90$ & $15.81 \%$ & 3 \\
\hline
\end{tabular}




\begin{tabular}{|c|c|c|c|c|}
\hline & wrist extensors & $23.21 \pm 3.61$ & $4.30 \%$ & 8 \\
\hline & trapezius muscle & $84.93 \pm 9.71$ & $15.74 \%$ & 4 \\
\hline & $\begin{array}{l}\text { latissimus dorsi } \\
\text { muscle }\end{array}$ & $96.92 \pm 10.82$ & $17.96 \%$ & 2 \\
\hline & $\begin{array}{c}\text { pectoralis major } \\
\text { muscle }\end{array}$ & $27.70 \pm 4.76$ & $5.13 \%$ & 7 \\
\hline \multirow{6}{*}{ Left } & rectus abdoninis & $55.86 \pm 5.65$ & $24.9 \%$ & 1 \\
\hline & gluteus maximus & $36.89 \pm 4.31$ & $16.44 \%$ & 3 \\
\hline & quadriceps & $29.97 \pm 3.32$ & $13.36 \%$ & 4 \\
\hline & hamstrings & $53.64 \pm 6.09$ & $23.91 \%$ & 2 \\
\hline & tibialis anterior & $20.41 \pm 2.53$ & $9.09 \%$ & 6 \\
\hline & gastrocnemius & $27.58 \pm 4.78$ & $12.29 \%$ & 5 \\
\hline
\end{tabular}

\section{DISCUSSION}

\subsection{Analysis on sEMG Characteristics in A-B Process in the Left Side of Muscle Groups: Deltoid Muscle, Biceps Brachii Muscle, Triceps Muscle of Arm, Wrist Flexors (Extensors), Trapezius Muscle, Latissimus Dorsi Muscle, and Pectoralis Major Muscle}

Analysis based on movement techniques, anatomical action analysis, and sEMG test results: at point $\mathrm{A}$ when the left hand of the subject began to hold the bridge post, at which time the arm in the shoulder joint around the frontal axis extended, latissimus dorsi muscle, posterior deltoid, long head of triceps, teres major, teres minor, and infraspinatus muscle were fixed to complete the concentric contraction; the forearm was bent around the frontal axis at the elbow joint, and biceps brachii muscle, brachialis, brachioradialis, and pronator teres were far fixed to complete concentric contraction; the hand was bent in the wrist around the frontal axis, the flexor carpi radialis, palmaris longus, flexor carpi ulnaris, flexor digitorum superficialis, and deep flexor muscle of finger were far fixed to complete concentric contraction. Original electromyography showed (Picture 3) in this process, deltoid muscle, biceps brachii muscle, triceps muscle of arm, wrist flexors, and latissimus dorsi muscle appeared obvious sEMG performance. The biceps brachii muscle, triceps muscle of arm, wrist flexors, latissimus dorsi muscle were the most active muscles, and maintained high-frequency large-scale vibration. It is suggested that relying on upper limb and shoulder-back force to get the body close to the bridge post in the process of curving the bridge post is a key technical movement to control inertial deviation. Subsequently, the left foot stepped out, and the upper body followed to move rapidly, with the body as close as possible to the bridge post along the shortest route; at this time in order to pull out the left arm as soon as possible, the shoulder blade dropped back, and the upper arm extended subconsciously. Original electromyography showed (Picture 3) in this process trapezius muscle and deltoid muscle appeared obvious sEMG performance, and trapezius muscle reached the peak. Combined with A-C segment $\mathrm{iEMG}$ and $\mathrm{iEMG \%}$ data analysis, the research showed that efforts should be made to develop the muscle strength of biceps brachii muscle, brachialis, triceps muscle of arm, wrist flexors, latissimus dorsi muscle, and trapezius muscle.

\subsection{Analysis on SEMG Characteristics in A-B Process in the Left Side of Muscle Groups: Rectus Abdoninis, Gluteus Maximus, Quadriceps, Hamstrings, Tibialis Anterior, and Gastrocnemius}

Analysis based on movement techniques, anatomical action analysis, and sEMG test results: in the A-B process, the subject bent down, and the left lower limb first completed the technical action of the forward step. At this time the thigh in the hip joint around the frontal axis bowed, and iliopsoas, rectus femoris, whip muscle, and muscle fascia expansion were near fixed to complete the concentric contraction; the calf is extended around the frontal axis at the knee joint, and the quadriceps near fixed to complete the concentric contraction; the foot was fixed at the ankle, and the calf front muscle and calf triceps were near fixed to complete isometric exercises. Original electromyography showed (Picture 3 ) in this process, the rectus abdoninis were the first to appear a high-frequency small vibration, and then the quadriceps, hamstrings, gastrocnemius, and tibialis anterior were activated in turn to complete forward movement. The original electromyography (Picture 3) showed that at the moment when the subject began to stepped out the left foot approaching point $\mathrm{B}$, there was significant sEMG performance in hamstrings and gastrocnemius, and the hamstrings presented larger sEMG vibration. Combined with A-B segment iEMG and iEMG\% dada analysis, the study showed that efforts should be made to develop the 
muscle strength in lower limb hamstrings, gluteus maximus, quadriceps, gastrocnemius and rectus abdoninis.

\section{CONCLUSIONS AND RECOMMENDATIONS}

In the technical action of bypassing bridge post, relying on upper limb and shoulder strength to make the body near the bridge body is the key technical link to control inertia deviation, and the main original muscle groups included upper limb biceps brachii muscle, brachialis, triceps muscle of arm, flexor forearm muscles, flexor muscle group and latissimus dorsi muscle; subsequently, the upper limb took a shortcut to quickly pull away from the bridge post, trapezius muscle and deltoid muscle as the main original muscles to complete subconsciously the technical action of shoulder contraction back and the upper arm extension.

Before the forward movement of the leg, the rectus abdoninis as the main original muscle completed the technical action of bending body, and then the quadriceps, calf front muscle and calf triceps as the main muscles completed the action of stepping out; hamstrings, gluteus maximus, quadriceps and calf triceps as the main original muscles completed the action of stretching and touching.

In the teaching and training of bypassing bridge post, great efforts should be made to develop the muscle strength of the upper limb biceps brachii muscle, brachialis, triceps muscle of arm, wrist flexors, latissimus dorsi muscle, and trapezius muscle; it was the main focus to develop the muscle strength of lower limb hamstrings, gluteus maximus, quadriceps, calf triceps and rectus abdoninis.

\section{REFERENCES}

[1] Lin Jianxuan, Bao Yuchun, Chen Nansheng, etc. Military Sports (Second Book) [M]. Beijing: National Defense Industry Press, 2012, 95-126.

[2] Li Yu. Application of Surface Myoelectricsy in Sports [M]. Shanghai: Fudan University Press, 2015, 59-60.

[3] Qiao Zhu. Study on Surface Electrocardiography Change Characteristics in Muscles of Increased Load Sprinters [J]. Journal of Shenyang Sports College, 2011, 30 (4): 78-81.

[4] Qu Feng. Athlete's Surface Muscle Electrical Signal and Fractals [M]. Beijing: Beijing Sports University Press, 2008, 4-5.

[5] Fan Hongbin, Sun Youping, Ji Liu. Study on Age and Gender Generality of Different Strength Index Based on Surface Myocardial Contribution Rate [J]. China Sports Technology, 2016, (52)5:83-97.
[6] Donath L,Kurz E,Roth R,et al.Different ankle muscle coordination patterns and co-activation during quiet stance between young adults and seniors do not change after a bout of high intensity training[J].BMC Geriatr,2015,15(1):19-27. 